\title{
Pulse radar randomly interrupted transmitting and receiving optimization based on genetic algorithm in radio frequency simulation
}

\author{
Xiaobin Liu* ${ }^{*}$, Feng Zhao ${ }^{*}$ Xiaofeng Ai and Qihua Wu
}

\author{
*Correspondence: xiaobinat08@ \\ yeah.net; zhfbee@tom.com \\ State Key Laboratory of Complex \\ Electromagnetic Environment \\ Effects on Electronics and \\ Information System, National \\ University of Defense Technology, \\ Changsha 410073, People's Republic \\ of China
}

\begin{abstract}
The interrupted transmitting and receiving (ITR) can be used in anechoic chamber to solve the coupling between the transmitted and reflected signals. When the ITR periods are random, the fake peaks in high-resolution range profile (HRRP) of ITR echo can be reduced. Then, by utilizing the piecewise sparse property of ITR echo, the HRRP is reconstructed based on compressive sensing (CS). However, the periods of ITR determine the restricted isometry property (RIP) condition and the HRRP reconstruction performance. In order to improve the HRRP reconstruction performance, the ITR period sequence optimization method based on a genetic algorithm (GA) is proposed in this paper. The correlation coefficient of the sensing matrix columns is minimized after optimization so that the RIP of the sensing matrix can be well satisfied. Simulation and comparison results illustrate that the optimization method converges fast and the HRRP reconstruction performance is improved with the optimized ITR periods.
\end{abstract}

Keywords: Pulse radar, Interrupted transmitting and receiving (ITR), Compressive sensing (CS), Genetic algorithm (GA)

\section{Springer Open}

\section{Introduction}

The radio frequency simulation (RFS) [1] in an anechoic chamber is widely adopted to conduct target measurement [2,3]. Because the size of an anechoic chamber is small, the round-trip time of electromagnetic wave may be sub-microsecond which is generally larger than the pulse width. Therefore, the reflected and transmitted signals may be coupled at the receiver when the pulse radar signal is applied in an anechoic chamber for target measurement. In order to solve the problem, the interrupted transmitting and receiving (ITR) method is proposed in [3]. The ITR can divide the pulse signal into hundreds of short pulses with each short pulse width being submicrosecond. As each short pulse width is smaller than the round-trip time, the coupling is eliminated and the target echo can be obtained for the target measurement.

(c) The Author(s). 2021, corrected publication 2021. Open Access This article is licensed under a Creative Commons Attribution 4.0 International License, which permits use, sharing, adaptation, distribution and reproduction in any medium or format, as long as you give appropriate credit to the original author(s) and the source, provide a link to the Creative Commons licence, and indicate if changes were made. The images or other third party material in this article are included in the article's Creative Commons licence, unless indicated otherwise in a credit line to the material. If material is not included in the article's Creative Commons licence and your intended use is not permitted by statutory regulation or exceeds the permitted use, you will need to obtain permission directly from the copyright holder. To view a copy of this licence, visit http://creativecommons.org/licenses/by/4.0/. 
During the ITR, part of the pulse signal is not transmitted, and the target highresolution range profile (HRRP) is difficult to be obtained with the ITR echo. An effective method is using compressive sensing (CS) [4-8] to reconstruct the HRRP. The sparse stepped-frequency signal is utilized in [5] to obtain the HRRP and target image with CS. The target HRRP estimation and target detection are discussed in [6-8] based on the sparse learning. By utilizing the property of ITR, the measurement matrix can be constructed according to the ITR period sequence and the HRRP is reconstructed [9].

However, the reconstruction performance of HRRP is generally determined by the restricted isometry property (RIP) condition of the sensing matrix [10-12]. Therefore, different sensing matrixes may result in different reconstruction performance. The eigenvalue of the Gram matrices and mutual coherence [11, 13] are mainly used to measure the RIP of the sensing matrix. In the ITR method, the sensing matrix is formed according to the ITR periods which means the ITR periods determine the RIP of the sensing matrix. Therefore, improving the HRRP reconstruction performance equals to optimizing the ITR period sequence.

Generally, the R-ITR period sequence is composed of multiple short pulse periods. And then, the optimization of period sequence becomes a multivariable optimization problem. Various multi-objective optimization methods, such as the genetic algorithm (GA) $[14,15]$ and the particle swarm optimization (PSO) [16, 17], have been investigated. But the algorithms are difficult to be used in optimizing the ITR period sequence directly. Therefore, the R-ITR period sequence optimization method is proposed based on GA in this paper. The minimization of the sensing matrix columns cross-correlation coefficient is used as the fitness criterion. Then, the R-ITR period sequence is optimized after an iteration, and the RIP of the sensing matrix can be better satisfied. The most important aspects of this paper are as follows:

1) The binary coded and decoded method for R-ITR period sequence is proposed so that the cross-operation and mutation operation can be performed during the optimization process.

2) The fitness function is formed for the optimization and the whole procedure of optimization is provided and discussed based on GA.

The remainder of this paper is organized as follows. In Section 2, we give a brief introduction to the principle of R-ITR for pulse radar. The property of ITR echo and the CS-based HRRP reconstruction are discussed as well. After that, the optimization method is proposed to design the R-ITR period sequence. In Section 3, simulations and comparison results are presented and discussed to verify the validity of the proposed optimization method. Finally, conclusions are drawn in Section 4.

\subsection{Notation}

In the sequel, vectors and matrixes are denoted by boldface. $[\cdot]^{\mathrm{T}}$ denotes the transpose operation. (.)* represents the conjugate operation. Symbol $\|\cdot\|$ denotes the Euclidean norm of a vector. $\min (\cdot)$ and $\max (\cdot)$ stand for the minimum and maximum operation. I stands for the identity matrix. A is the sensing matrix. $\Psi$ is the $N \times N$ inverse fast 
Fourier transform matrix. $\boldsymbol{\Phi}$ is the measurement matrix. $\rho(\cdot)$ is the maximum crosscorrelation coefficient of the sensing matrix. $F(\cdot)$ is the fitness function in the optimization method.

\section{Methods}

\subsection{Principle of R-ITR in anechoic chamber}

In an anechoic chamber, the pulse signal is divided into multiple short pulses after ITR so that the target echo can be received when the next short pulse is transmitted. Then, the coupling can be eliminated [3]. When the ITR periods are random, the R-ITR is shown in Fig. 1.

The R-ITR control signal can be expressed as:

$$
p_{1}(t)=\sum_{n \rightarrow-\infty}^{+\infty} \operatorname{rect}\left(\frac{t}{\tau_{n}}\right) * \delta\left(t-\sum_{k}^{n} T_{s_{k}}\right)
$$

where $\delta(\cdot)$ is the impulse function and $\tau_{n}$ is the width of the $n$th short pulse. $T_{s_{k}}$ is the period of the $k$ th short pulse and $k \leq n$. * is the convolution operation, and $\operatorname{rect}(\cdot)$ is:

$$
\operatorname{rect}\left(\frac{t}{\tau_{n}}\right)=\left\{\begin{array}{c}
1,\left|t / \tau_{n}\right|<0.5 \\
0, \text { others }
\end{array}\right.
$$

When the period of ITR and short pulse width is unchanged and it can suppose as $T_{s_{k}}=T_{s}$ and $\tau_{n}=\tau$, then we have $\sum_{k}^{n} T_{s_{k}}=n T_{s}$ for the $n$th short pulse. And the uniform ITR control signal is:

$$
p_{2}(t)=\operatorname{rect}\left(\frac{t}{\tau}\right) * \sum_{n \rightarrow-\infty}^{+\infty} \delta\left(t-n T_{s}\right)
$$

Therefore, the spectrum of $p_{2}(t)$ can be obtained as:

$$
P_{2}(f)=\tau f_{s} \sum_{n=-\infty}^{n=+\infty} \operatorname{sinc}\left(n f_{s} \tau\right) \delta\left(f-n f_{s}\right)
$$

where $f_{s}=1 / T_{s}, \sin c(x)=\sin (\pi x) /(\pi x)$. As the R-ITR period is random, the analytical expression of the spectrum is difficult to be obtained.

The transmitted signal is supposed as linear frequency modulation (LFM)

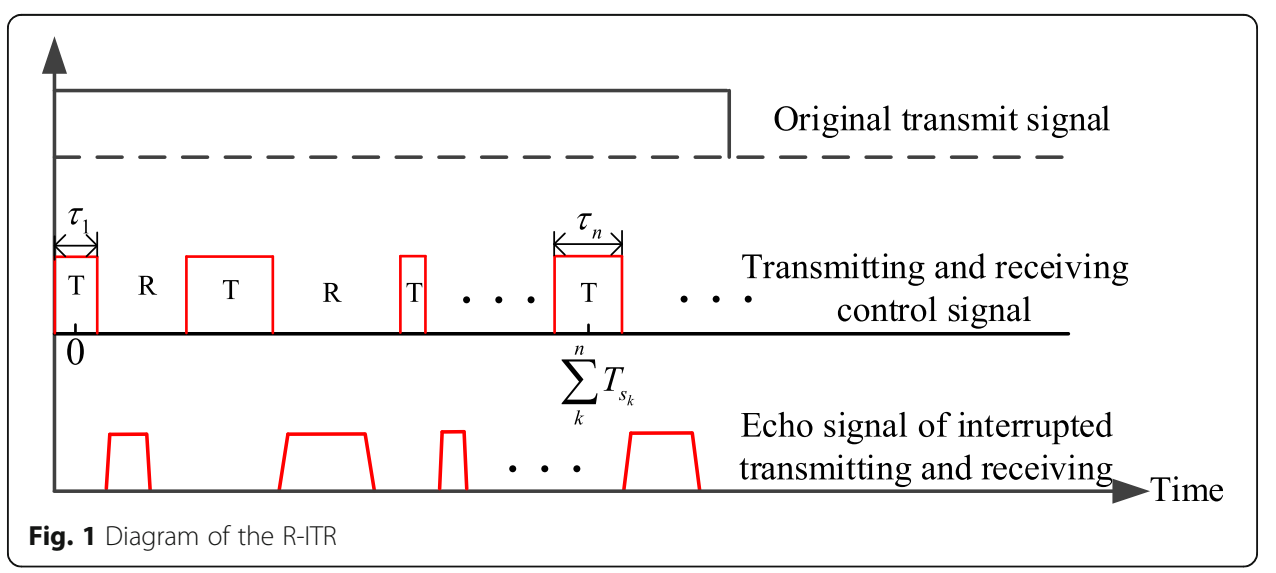




$$
s(t)=\operatorname{rect}\left(\frac{t}{T_{p}}\right) \exp \left(j 2 \pi\left(f_{c} t+\frac{1}{2} \mu t^{2}\right)\right)
$$

where $T_{p}$ is the pulse width, $f_{c}$ is the carrier frequency, and $\mu=B / T_{p}$ and $B$ are the bandwidth.

Considering the ITR control signal in (3), the ITR echo can be obtained as:

$$
\begin{array}{r}
s_{r_{2}}(t)=p_{2}(t) \cdot s_{r}(t) \\
=\left(\operatorname{rect}\left(\frac{t}{\tau}\right) * \sum_{n \rightarrow-\infty}^{+\infty} \delta\left(t-n T_{s}\right)\right) s_{r}(t)
\end{array}
$$

where $s_{r}(t)=\sum^{K} \alpha_{k} s\left(t-2 R_{k} / C\right)$ is the target echo, $K$ is the number of scattering centers, and $\alpha_{k}$ is $k_{\bar{h}}^{1}$ e scattering coefficient of the $k$ th scattering centers. $R_{k}$ is the distance between the radar and the $k$ th scattering center.

The de-chirp reference signal is $s_{\text {ref }}(t)=s\left(t-2 R_{\text {ref }} / C\right)$, where $R_{\text {ref }}$ is the reference distance and $C$ is the speed of the electromagnetic wave. Then, the difference output after de-chirping is:

$$
\begin{aligned}
& s_{f_{2}}(t)=p_{2}(t) \cdot s_{r}(t) \cdot s_{\mathrm{ref}}^{*}(t) \\
& =\sum_{k=1}^{K}\left(\left(\operatorname{rect}\left(\frac{t}{\tau}\right) * \sum_{n \rightarrow-\infty}^{+\infty} \delta\left(t-n T_{s}\right)\right) \alpha_{k} \operatorname{rect}\left(\frac{t-2 R_{k} / C}{T_{p}}\right) \exp \left(-j \frac{4 \pi}{C} \mu\left(t-\frac{2 R_{\mathrm{ref}}}{C}\right) R_{k, \Delta}\right)\right. \\
& \left.\quad \cdot \exp \left(-j \frac{4 \pi}{C} f_{c} R_{k, \Delta}\right) \exp \left(j \frac{4 \pi}{C^{2}} R_{k, \Delta}^{2}\right)\right)
\end{aligned}
$$

where $s_{\text {ref }}^{*}(t)$ is the conjugation of $s_{\text {ref }}(t)$, and $R_{k, \Delta}=R_{k}-R_{\text {ref }}$ is the distance between the scattering center and the reference point.

The HRRP of ITR echo is obtained after Fourier transform:

$$
S_{f_{2}}(f)=\tau f_{s} T_{p} \sum_{k=1}^{K} \alpha_{k} \exp \left(-j \frac{4 \pi f_{c}}{C} R_{k, \Delta}\right) \sum_{n \rightarrow-\infty}^{n \rightarrow+\infty}\left(\operatorname{sinc}\left(n f_{s} \tau\right) \operatorname{sinc}\left(T_{p}\left(f-n f_{s}+2 \frac{\mu}{C} R_{k, \Delta}\right)\right)\right)
$$

In Eq. (8), $n f_{s}$ denotes that different orders of fake peaks appear in HRRP. Then, the range interval is:

$$
\Delta R=\frac{C f_{s}}{2 \mu}
$$

The constraints of ITR parameters are the same with [9]:

$$
\left\{\begin{array}{l}
\tau \leq \frac{2 R_{0}}{C} \\
\tau+\frac{2\left(R_{0}+L\right)}{C} \leq T_{s}<\frac{C T_{p}}{2 B L}
\end{array}\right.
$$

Because $\Delta R$ may be smaller than $L$, the real and fake peaks will be overlapped and the target peaks in the HRRP are difficult to be extracted. However, the ITR echo is sparse compared with the completed pulse; therefore, the HRRP can be reconstructed based on CS.

\subsection{HRRP reconstruction based on CS}

The vector of the difference-frequency output of the complete echo can be expressed as: 


$$
\mathbf{s}_{\mathbf{f}}=\mathbf{S}_{\mathbf{r e f}}^{*} \mathbf{s}_{\mathbf{r}}
$$

where $\mathbf{s}_{\mathbf{r}}=\left[s_{r}(0), s_{r}(1), \ldots, s_{r}(N-1)\right]^{T}$ is $N \times 1$ vector of the complete echo $s_{r}(t), N$ is the total sampling number in $T_{p}, \mathbf{S}_{\text {ref }}=\operatorname{diag}\left\{s_{\text {ref }}(0), s_{\text {ref }}(1), \ldots, S_{\text {ref }}(N-1)\right\}$ is the $N \times N$ diagonal matrix composed by the reference signal $s_{\text {ref }}(t)$, and $\mathbf{s}_{\mathbf{f}}=\left[s_{f}(0), s_{f}(1), \ldots, s_{f}(N-1)\right]^{T}$ is the $N$ $\times 1$ vector of the difference-frequency output.

$\mathbf{S}_{\mathbf{f}}=\left[S_{f}(0), S_{f}(1), \ldots, S_{f}(N-1)\right]^{T}$ is the target HRRP which can be supposed as $K$-sparse according to the number of scattering centers. Then, $\mathbf{S}_{\mathbf{f}}$ can be obtained after Fourier transform of (11):

$$
\mathbf{S}_{\mathbf{f}}=\Psi^{-1} \mathbf{S}_{\mathrm{ref}}^{*} \mathbf{S}_{\mathbf{r}}
$$

where $\boldsymbol{\Psi}$ is the $N \times N$ inverse fast Fourier transform (IFFT) matrix.

Because $p_{1}(t)$ is 1 in $\tau_{n}$ and 0 in $T_{S_{n}}-\tau_{n}$, the rows of $\mathbf{P}=\operatorname{diag}\left\{p_{1}(0), p_{1}(1), \ldots, p_{1}(N-1)\right\}$ are 0 and can be eliminated. Then, the formation of $\mathbf{P}_{1}$ is obtained and presented in Fig. 2.

$\mathbf{P}_{1}$ can be expressed as:

$$
\mathbf{P}_{1}=\left(\begin{array}{cccccccc}
\mathbf{I}_{n_{s_{1}}} & 0 & \cdots & 0 & 0 & \cdots & 0 & 0 \\
0 & 0 & \cdots & 0 & \mathbf{I}_{s_{s_{2}}} & \cdots & 0 & 0 \\
\vdots & \vdots & \cdots & \vdots & \vdots & \cdots & \vdots & \vdots \\
0 & 0 & \cdots & 0 & 0 & \cdots & 0 & \mathbf{I}_{n_{s_{n}}^{\prime}}
\end{array}\right)_{M \times N}
$$

where $\mathbf{I}_{n s n}$ is the $n_{s n} \times n_{s n}$ identity matrix.

Then, the ITR echo is obtained based on the measurement of $\mathbf{s}_{\mathbf{r}}$ :

$$
\mathbf{s}_{\mathrm{TTR}}=\mathbf{P}_{1} \mathbf{s}_{\mathbf{r}}
$$

where $\mathbf{S}_{\text {ITR }}$ is the $M \times 1$ vector.

As $\mathbf{S}_{\text {ref }} \mathbf{S}_{\text {ref }}^{*}=\mathbf{I}$, Eq. (14) can be re-written as:

$$
\mathbf{s}_{\mathrm{ITR}}=\mathbf{P}_{1} \mathbf{S}_{\mathrm{ref}} \Psi \mathbf{S}_{\mathbf{f}}
$$

After adding noise $\boldsymbol{\xi}$, we have:

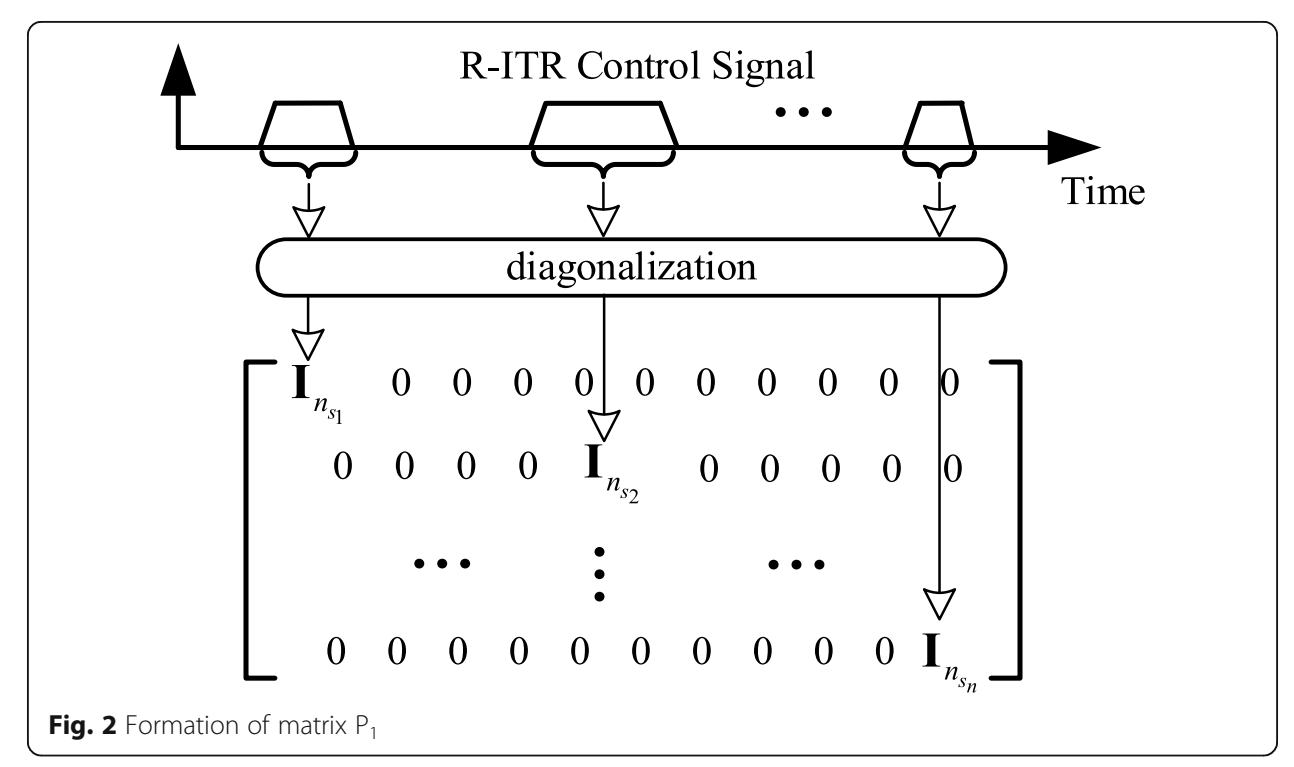




$$
\begin{aligned}
\mathbf{s}_{\text {spInter }}= & \mathbf{s}_{\mathrm{ITR}}+\boldsymbol{\xi} \\
& =\boldsymbol{\Phi} \boldsymbol{\Psi} \mathbf{S}_{\mathbf{f}}+\boldsymbol{\xi}
\end{aligned}
$$

where $\boldsymbol{\Phi}=\mathbf{P}_{1} \mathbf{S}_{\text {ref }}$ and $\mathbf{s}_{\text {spInter }}$ is the non-zero value of ITR echo.

The target HRRP can be reconstructed by solving the following problem:

$$
\min _{\overline{\mathbf{S}_{\mathbf{f}}}}\left\|\overline{\mathbf{S}_{\mathbf{f}}}\right\|_{1} \quad \text { s.t. }\left\|\mathbf{s}_{\text {spInter }}-\boldsymbol{\Phi} \Psi \overline{\mathbf{S}_{\mathbf{f}}}\right\|_{2} \leq \boldsymbol{\xi}
$$

where $\|\cdot\|_{1}$ is the $\ell_{1}$ norm, and $\overline{\mathbf{S}_{\mathbf{f}}}$ denotes the reconstructed HRRP.

Let $\mathbf{A}=\boldsymbol{\Phi} \Psi$ be the sensing matrix. $\Psi$ is the $N \times N$ the fixed IFFT matrix. $\boldsymbol{\Phi}=$ $\mathbf{P}_{1} \mathbf{S}_{\text {ref }}$ is the measurement matrix which can be obtained with $\mathbf{P}_{1}$ and $\mathbf{S}_{\mathbf{r e f}}$. Because $\mathbf{S}_{\text {ref }}$ is composed by the reference signal $s_{\text {ref }}(t)$, the form of $\mathbf{A}$ is determined by the matrix $\mathbf{P}_{1}$. From Fig. 2, it can be found that different ITR period sequences can obtain different matrixes of $\mathbf{P}_{1}$ so that the form of $\mathbf{A}$ changes. Therefore, the relationship between the R-ITR period sequence and RIP conditions of $\mathbf{A}$ is constructed. Generally, A should satisfy the $K$-order RIP condition to ensure the HRRP reconstruction performance. In order to improve the RIP condition of $\mathbf{A}$, the R-ITR period sequence should be optimized.

\subsection{ITR period sequence optimization based on GA}

Because there are hundreds of short pulses in each pulse signal, the R-ITR period sequence optimization can be converted to a multivariable optimization problem. GA is an adaptive optimization algorithm which is widely used to solve the multivariable optimization problem. The multi-point search, cross-operation, and mutation operation are adopted to eliminate the worst solution and save the optimum solution. After iteration, the optimum R-ITR period sequence can be obtained.

According to Fig. 3, the R-ITR period should be coded firstly to obtain the initialized population. Supposing the R-ITR period $T_{S_{n}}$ is distributed in [0.5 $\left.\mu \mathrm{s}, 0.8 \mu \mathrm{s}\right]$ with the interval of $0.1 \mu \mathrm{s}$, there are four values to choose, i.e., $0.5 \mu \mathrm{s}, 0.6 \mu \mathrm{s}, 0.7 \mu \mathrm{s}$, and $0.8 \mu \mathrm{s}$. So, the two-bit binary can be adopted to code $T_{s_{n}}$, i.e., $00,01,10$, and 11 . $\mathbf{T}=\left\{T_{s_{n}, m^{\prime}}\right\}$ is a $N^{\prime} \times M^{\prime}$ matrix where $N^{\prime}$ is the number of $T_{S_{n}}$ and $M^{\prime}$ is the number of population. If the $m$ th R-ITR period sequence $T_{s_{n}, m^{\prime}}$ is:

$$
0.8 \mu \mathrm{s}, 0.6 \mu \mathrm{s}, 0.5 \mu \mathrm{s}, 0.6 \mu \mathrm{s}, 0.7 \mu \mathrm{s}, 0.7 \mu \mathrm{s}
$$

The corresponding binary codes $Z_{n, m^{\prime}}$ are obtained as:

$$
110100011010
$$

Generally, the sequence length of R-ITR periods is determined by the pulse width and the range of ITR periods. Therefore, the code length increases when the pulse width is large.

Researches show that the RIP of the sensing matrix equals to the eigenvalues of the Gram matrix of sensing matrix and the cross-correlation coefficient of the sensing matrix columns [11-13]. When the eigenvalues of the Gram matrix of sensing matrix are close to 1, the RIP property can be satisfied well. However, as the computation complexity of the Gram matrix eigenvalues is high, the cross- 


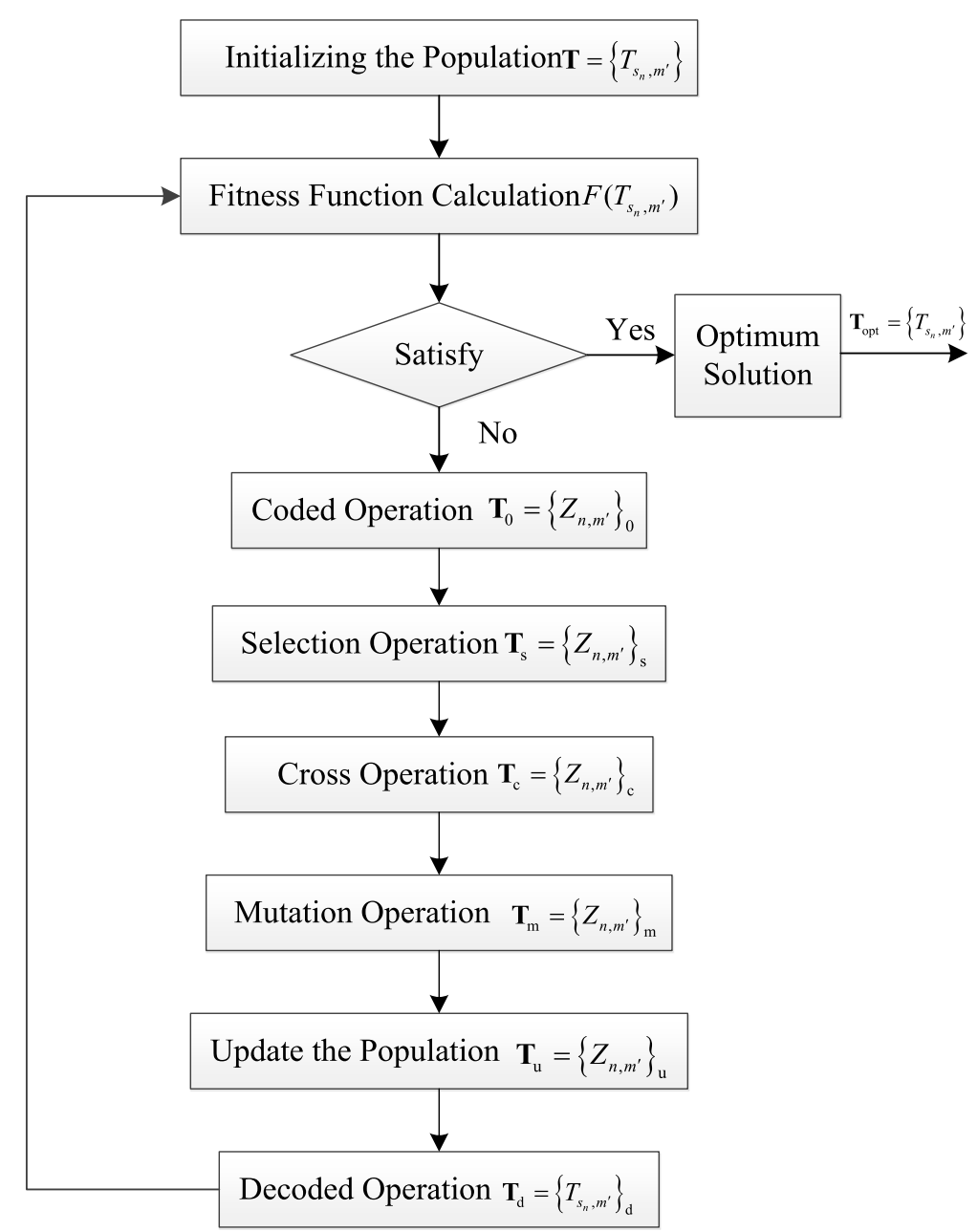

Fig. 3 R-ITR period sequence optimization based on GA

correlation coefficient of the sensing matrix columns is adopted to evaluate the fitness of the population.

$\mathbf{a}_{i}$ is supposed as the $i$ th column of the sensing matrix A. Then, the maximum crosscorrelation coefficient [11-13] of the sensing matrix $\mathbf{A}$ is defined as:

$$
\rho(\mathbf{A})=\max _{0 \leq k, j<N, k \neq j} \frac{\left|\mathbf{a}_{k}^{H} \mathbf{a}_{j}\right|}{\left\|\mathbf{a}_{k}^{H}\right\|_{2}\left\|\mathbf{a}_{j}\right\|_{2}}
$$

When the maximum cross-correlation coefficient is minimized, the RIP of the sensing matrix can be well satisfied. Therefore, we define the fitness function with the $m$ th RITR period sequence $T_{s_{n}, m^{\prime}}$ as:

$$
F\left(T_{s_{n}, m^{\prime}}\right)=\max \frac{1}{\rho(\mathbf{A})}, \quad \text { s.t. } \quad T_{s_{n}, m^{\prime}} \in P
$$

where $P$ is the variation range of $T_{S_{n}}$.

The scheme of the proposed optimization method based on GA is presented in Fig. 3.

The flow of Algorithm 1 is presented according to Fig. 3. 
Algorithm 1: R-ITR periods sequence optimization.

Input: Initial population $\mathbf{T}=\left\{T_{s_{n}, m^{\prime}}\right\}$

Output: Optimized R-ITR periods sequence $\mathbf{T}_{\mathrm{opt}}=\left\{T_{s_{0}, m^{\prime}}\right\}$

1. Calculate $F\left(T_{s_{n}, m^{\prime}}\right)$

2. Judge the fitness value

3. Code the R-ITR periods sequence to be $\mathbf{T}_{0}=\left\{Z_{n, m^{\prime}}\right\}_{0}$

4. Select the optimum individual $\mathbf{T}_{\mathrm{s}}=\left\{Z_{n, m^{\prime}}\right\}_{\mathrm{s}}$

5. Cross operation and obtain $\mathbf{T}_{c}=\left\{Z_{n, m^{\prime}}\right\}_{c}$

6. Mutation operation and obtain $\mathbf{T}_{m}=\left\{Z_{n, m^{\prime}}\right\}_{m}$

7. Population update and obtain $\mathbf{T}_{\mathrm{u}}=\left\{Z_{n, m^{\prime}}\right\}_{m}$

8. Decode operation to obtain $\mathbf{T}_{\mathrm{d}}=\left\{T_{s_{\mathrm{n}}, m^{\prime}}\right\}_{\mathrm{d}}$ and return to 1

By initializing the population as $\mathbf{T}=\left\{T_{s_{n}, m^{\prime}}\right\}$, the proposed optimization process is expressed in detail according to Algorithm 1 and Fig. 3.

Step 1. Calculate the fitness function. The sensing matrix $A=\Phi \Psi$ is constructed based on each individual $T_{s_{n}, m^{\prime}}$. Then, the fitness function in Eq. (21) is calculated.

Step 2. Judge the fitness value. The fitness function is calculated for every R-ITR period sequence. If the fitness value is satisfied, the iteration stops. Otherwise, the iteration continues.

Step 3. Code operation. The length of the binary code is determined by the range of the R-ITR periods. According to the coded principle in Eqs. (18) and (19), the R-ITR period sequence can be coded as $\mathbf{T}_{0}=\left\{Z_{n, m^{\prime}}\right\}_{0} . M^{\prime}$ series R-ITR period sequences are generated in this step.

Step 4. Select the optimum individual. The roulette wheel is used to select the individual. The elitism selection is also adopted in this part to save the optimum individual $\left\{Z_{n, m}\right\}$.

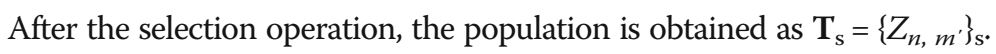

Step 5. Cross operation. The two-point crossover is utilized to conduct the cross operation. The crossover positions and individuals are randomly selected with the crossover probability of $P_{c}$. Then, the new individuals can be obtained. And the updated population is $\mathbf{T}_{\mathrm{c}}=\left\{Z_{n, m^{\prime}}\right\}_{\mathrm{c}}$.

Step 6. Mutation operation. The mutation operation is conducted based on the population obtained in step 5 . The single-point mutation is performed by randomly selecting the individual and the mutation position with the mutation probability of $P_{m}$. Then, the population after mutation operation is $\mathbf{T}_{\mathrm{m}}=\left\{Z_{n, m^{\prime}}\right\}_{\mathrm{m}}$.

Step 7. Population update. The elitism selection is still used to update the population and obtain $\mathbf{T}_{\mathrm{u}}=\left\{Z_{n, m^{\prime}}\right\}_{\mathrm{u}}$.

Table 1 The R-ITR period sequence before and after optimization $\left(T_{S_{n}} \in[0.5 \mu \mathrm{s}, 0.8 \mu \mathrm{s}]\right)$

\begin{tabular}{llllllllllll}
\hline Before & $\mathbf{0 . 8}$ & $\mathbf{0 . 8}$ & $\mathbf{0 . 8}$ & $\mathbf{0 . 5}$ & $\mathbf{0 . 7}$ & $\mathbf{0 . 7}$ & $\mathbf{0 . 8}$ & $\mathbf{0 . 7}$ & $\mathbf{0 . 6}$ & $\mathbf{0 . 7}$ & $\mathbf{0 . 8}$ \\
optimization & $\mathbf{0 . 7}$ & $\mathbf{0 . 5}$ & $\mathbf{0 . 8}$ & $\mathbf{0 . 5}$ & $\mathbf{0 . 7}$ & $\mathbf{0 . 5}$ & $\mathbf{0 . 6}$ & $\mathbf{0 . 8}$ & $\mathbf{0 . 5}$ & $\mathbf{0 . 8}$ & $\mathbf{0 . 7}$ \\
& $\mathbf{0 . 6}$ & $\mathbf{0 . 5}$ & $\mathbf{0 . 6}$ & $\mathbf{0 . 8}$ & $\mathbf{0 . 5}$ & $\mathbf{0 . 7}$ & $\mathbf{0 . 7}$ & $\mathbf{0 . 7}$ & & & \\
\hline After optimization & 0.7 & 0.8 & 0.5 & 0.5 & 0.8 & 0.8 & 0.8 & 0.5 & 0.5 & 0.8 & 0.7 \\
& 0.7 & 0.8 & 0.5 & 0.8 & 0.5 & 0.5 & 0.6 & 0.6 & 0.5 & 0.5 & 0.5 \\
& 0.5 & 0.8 & 0.8 & 0.5 & 0.5 & 0.5 & 0.8 & 0.7 & 0.5 & 0.5 & 0.8 \\
\hline
\end{tabular}




\begin{tabular}{lccccccccc} 
Table 2 The R-ITR period sequence before and after optimization $\left(T_{s_{n}} \in[0.5 \mu \mathrm{s}, 1.2 \mu \mathrm{s}]\right.$ & \\
\hline Before & $\mathbf{0 . 9}$ & $\mathbf{1 . 2}$ & $\mathbf{1 . 0}$ & $\mathbf{0 . 7}$ & $\mathbf{1 . 0}$ & $\mathbf{1 . 0}$ & $\mathbf{1 . 2}$ & $\mathbf{1 . 1}$ & $\mathbf{0 . 9}$ \\
optimization & $\mathbf{0 . 9}$ & $\mathbf{1 . 2}$ & $\mathbf{1 . 0}$ & $\mathbf{0 . 5}$ & $\mathbf{0 . 7}$ & $\mathbf{0 . 8}$ & $\mathbf{0 . 6}$ & $\mathbf{1 . 1}$ & $\mathbf{1 . 1}$ \\
& $\mathbf{0 . 5}$ & $\mathbf{0 . 6}$ & $\mathbf{0 . 6}$ & $\mathbf{0 . 8}$ & $\mathbf{0 . 9}$ & & & & \\
\hline After optimization & 0.5 & 0.7 & 0.6 & 1.1 & 0.6 & 1.0 & 0.8 & 0.5 & 0.8 \\
& 1.2 & 1.2 & 1.2 & 0.5 & 0.8 & 0.5 & 0.8 & 0.5 & 0.9 \\
& 1.0 & 1.1 & 1.0 & 0.5 & 0.8 & 0.7 & 1.0 & 0.9 & \\
\hline
\end{tabular}

Step 8. Decode operation. As $\mathbf{T}_{\mathrm{u}}=\left\{Z_{n, m^{\prime}}\right\}_{\mathrm{u}}$ is a binary code, $\mathbf{T}_{\mathrm{u}}$ should be decoded to obtain the R-ITR periods as $\mathbf{T}_{\mathrm{d}}=\left\{T_{s_{n}, m^{\prime}}\right\}_{\mathrm{d}}$. Then, $\mathbf{T}_{\mathrm{d}}$ is used to calculate the fitness function in step 1 .

\section{Results and discussion}

\subsection{Optimization results of the cross-correlation coefficient}

The pulse width is $T_{p}=20 \mu$ s with a bandwidth of $B=500 \mathrm{MHz}$. The distance between the radar and target is $R=45 \mathrm{~m}$. The R-ITR period sequences are set as $0.5 \sim 0.8 \mu \mathrm{s}$, $0.5 \sim 1.2 \mu \mathrm{s}$, and $0.8 \sim 1.1 \mu \mathrm{s}$ with an interval of $0.1 \mu \mathrm{s}$. The $\tau / f_{s}$ is 0.25 so that the maximum short pulse width is $0.3 \mu$ s which ensures the target echo can be received according to Eq. (10).

When $T_{s_{n}}$ distributes in $[0.5 \mu \mathrm{s}, 0.8 \mu \mathrm{s}]$ and $[0.8 \mu \mathrm{s}, 1.1 \mu \mathrm{s}]$, the two-bit binary can be used to code the R-ITR period sequence. When $T_{s_{n}}$ distributes in $[0.5 \mu \mathrm{s}, 1.2 \mu \mathrm{s}]$, there are 8 kinds of R-ITR periods. Therefore, three-bit binary should be used to code the $T_{s_{n}}$. The cross-probability and mutation probability are $P_{c}=0.57$ and $P_{m}=0.002$. The initialization population is $M^{\prime}=75$.

The length of the R-ITR period sequence is different according to the pulse width and $T_{s_{n}}$. Thus, the fitness function is calculated by choosing the front $20 \mu \mathrm{s}$. Then, the R-ITR periods are adopted to perform the optimization algorithm. According to the steps discussed in Section 2, the optimization results are presented in Tables 1, 2, and 3.

According to Eq. (21), the maximum fitness function value corresponds to the minimum correlation coefficient. The maximum value and average value of the fitness function versus the iteration are shown in Fig. 4.

Figure $4 \mathrm{a}$ is the maximum value of the fitness function versus the iteration. When the iterations are less, the maximum fitness function value of $T_{s_{n}} \sim[0.5 \mu \mathrm{s}, 1.2 \mu \mathrm{s}]$ is bigger than that of $T_{s_{n}} \sim[0.5 \mu \mathrm{s}, 0.8 \mu \mathrm{s}]$. The reason is that there are many individuals containing small $T_{s_{n}}$ in the initialized population when the R-ITR period range is $T_{s_{n}}$

Table 3 The R-ITR period sequence before and after optimization $\left(T_{s_{n}} \in[0.8 \mu \mathrm{s}, 1.1 \mu \mathrm{s}]\right)$

\begin{tabular}{lllllllll}
\hline Before & $\mathbf{0 . 9}$ & $\mathbf{0 . 9}$ & $\mathbf{0 . 8}$ & $\mathbf{1 . 0}$ & $\mathbf{0 . 9}$ & $\mathbf{0 . 9}$ & $\mathbf{1 . 1}$ & $\mathbf{1 . 0}$ \\
& $\mathbf{0 . 8}$ & $\mathbf{0 . 9}$ & $\mathbf{1 . 0}$ & $\mathbf{1 . 0}$ & $\mathbf{1 . 1}$ & $\mathbf{1 . 0}$ & $\mathbf{0 . 9}$ & $\mathbf{1 . 0}$ \\
& $\mathbf{1 . 0}$ & $\mathbf{1 . 0}$ & $\mathbf{1 . 1}$ & $\mathbf{0 . 9}$ & $\mathbf{0 . 9}$ & $\mathbf{0 . 8}$ & & \\
\hline After optimization & 0.8 & 0.8 & 0.8 & 0.8 & 0.8 & 1.1 & 0.8 & 0.8 \\
& 1.1 & 0.8 & 0.9 & 1.0 & 0.8 & 0.9 & 0.9 & 1.0 \\
& 1.1 & 1.1 & 1.1 & 1.1 & 1.1 & 0.9 & & \\
\hline
\end{tabular}




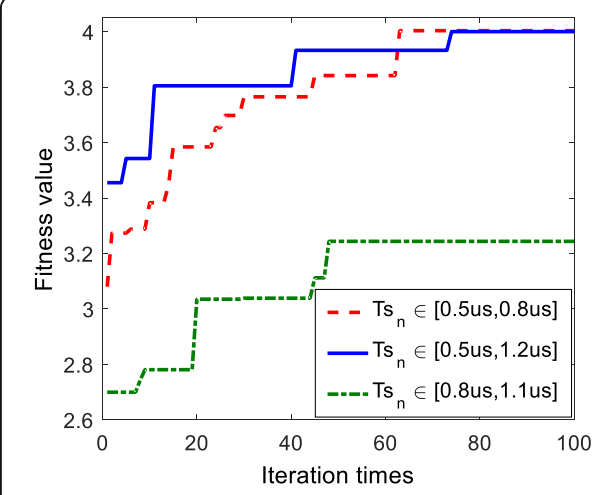

(a)

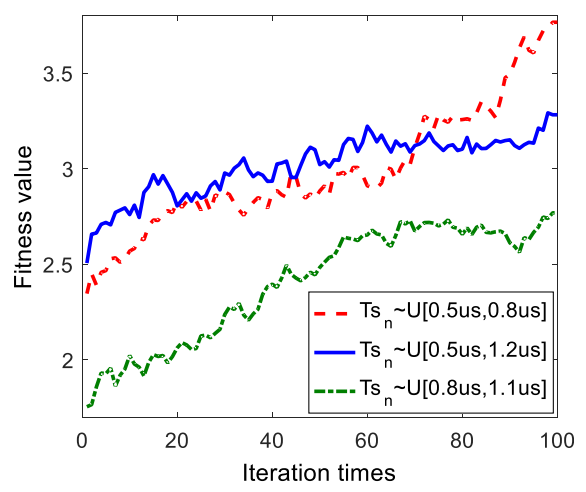

(b)

Fig. 4 The maximum and average values of the fitness function versus the iteration. a The maximum value of the fitness function versus the iteration. $\mathbf{b}$ The average value of the fitness function versus the iteration

$\sim[0.5 \mu \mathrm{s}, 1.2 \mu \mathrm{s}]$. However, the initialized population is not the optimized solution. When the iterations increase, the fitness function value of $T_{S_{n}} \sim[0.5 \mu \mathrm{s}, 0.8 \mu \mathrm{s}]$ increases and becomes bigger than that of $T_{s_{n}} \sim[0.5 \mu \mathrm{s}, 1.2 \mu \mathrm{s}]$. Besides, the maximum value of the fitness value is the smallest when $T_{s_{n}} \sim[0.8 \mu \mathrm{s}, 1.1 \mu \mathrm{s}]$.

The average value of the fitness function is presented in Fig. $4 \mathrm{~b}$. It can be found the curve tendency is the same with the maximum value of the fitness function in Fig. 4a. As the worst individuals are eliminated after iteration, the fitness function value increases so that the columns cross-correlation coefficient of the sensing matrix decreases. Then, the RIP of sensing matrix can be better satisfied after GA optimization.

In order to illustrate the columns cross-correlation coefficient variation of the sensing matrix, the average columns cross-correlation coefficient is obtained to compare with the coefficient after optimization. The columns cross-correlation coefficient of the proposed method is compared with the particle swarm optimization (PSO) algorithm. The results are shown in Table 4.

The optimization value in Table 4 is obtained according to Eq. (21). It can be found that the columns cross-correlation coefficient of the initialization individuals is 0.4131 when $T_{s_{n}} \sim[0.5 \mu \mathrm{s}, 0.8 \mu \mathrm{s}]$ and 0.4085 when $T_{s_{n}} \sim[0.5 \mu \mathrm{s}, 1.2 \mu \mathrm{s}]$. The cross-correlation coefficient is the maximum when $T_{S_{n}} \sim[0.8 \mu \mathrm{s}, 1.1 \mu \mathrm{s}]$. The results are basically identical with the curves in Fig. 4.

On the other hand, the columns cross-correlation coefficients are decreased after the optimization, i.e., $0.2498,0.2500$, and 0.3083 . Obviously, the coefficient of $T_{s_{n}} \sim[0.5 \mu \mathrm{s}$, $0.8 \mu \mathrm{s}$ ] is the minimum. Besides, the columns cross-correlation coefficient obtained by the PSO method is larger than that obtained by the proposed method. The reason is

Table 4 The columns of cross-correlation coefficient of the sensing matrix before and after optimization

\begin{tabular}{llll}
\hline & $T_{s_{n}} \in[0.5 \mu \mathrm{s}, 0.8 \mu \mathrm{s}]$ & $T_{S_{n}} \in[0.5 \mu \mathrm{s}, 1.2 \mu \mathrm{s}]$ & $T_{s_{n}} \in[0.8 \mu \mathrm{s}, 1.1 \mu \mathrm{s}]$ \\
\hline Before optimization & 0.4131 & 0.4085 & 0.5869 \\
PSO-based optimization method & 0.2590 & 0.2659 & 0.3398 \\
Proposed optimization method & 0.2498 & 0.2500 & 0.3083 \\
\hline
\end{tabular}




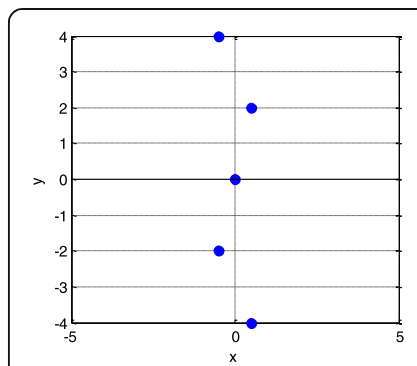

(a)

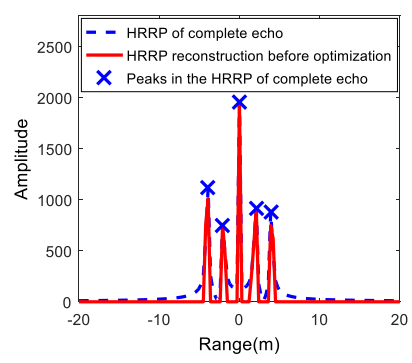

(b)

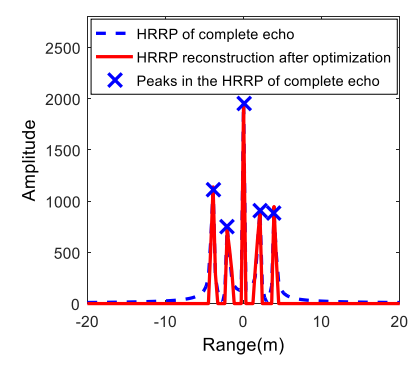

(c)

Fig. 5 The HRRP reconstruction comparison when $T_{s_{n}} \sim[0.5 \mu \mathrm{s}, 0.8 \mu \mathrm{s}]$. a Distribution of the scattering centers. $\mathbf{b}$ HRRP reconstruction before optimization. $\mathbf{c}$ HRRP reconstruction after optimization

that the proposed method adopts the elitism selection to save the best individual during each iteration. As a result, the RIP of the sensing matrix can be better satisfied with the proposed optimization method. And finally, the HRRP reconstruction performance can be improved.

\subsection{Target HRRP reconstruction results based on GA}

In this part, the HRRP reconstruction is conducted based on different R-ITR period sequence. Considering there are five scattering centers in the target, the distance between the scattering centers is $2 \mathrm{~m}$. The scattering coefficient is $[0.7,0.5$, $1,0.6,0.55]$. Firstly, the reconstructed HRRP is obtained when $T_{S_{n}} \sim[0.5 \mu \mathrm{s}, 0.8 \mu \mathrm{s}]$. Secondly, the R-ITR period sequence is optimized based on the proposed optimization method. The HRRP reconstruction after optimization is obtained, and the results are presented in Fig. 5.

Figure $5 \mathrm{a}$ is the distribution of target scattering centers. The scattering centers from -4 to $4 \mathrm{~m}$ is supposed as 1 to 5 . Figure $5 \mathrm{~b}$, c is the HRRP reconstruction before and after optimization. It can be found that the reconstructed HRRPs are basically identical to the HRRP of complete echo. Therefore, the sensing matrixes obtained by the

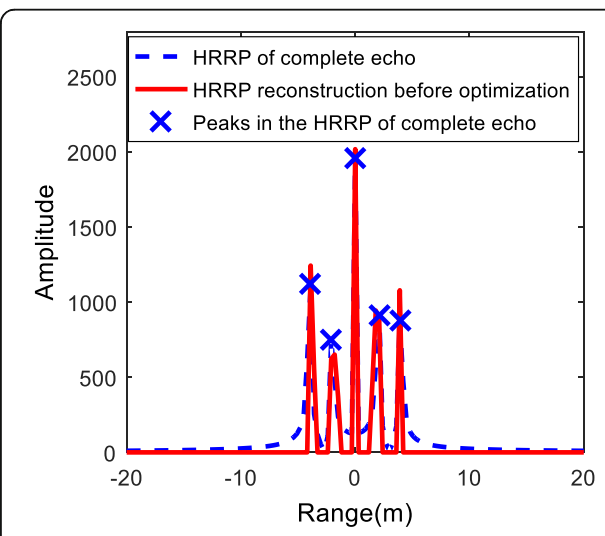

(a)

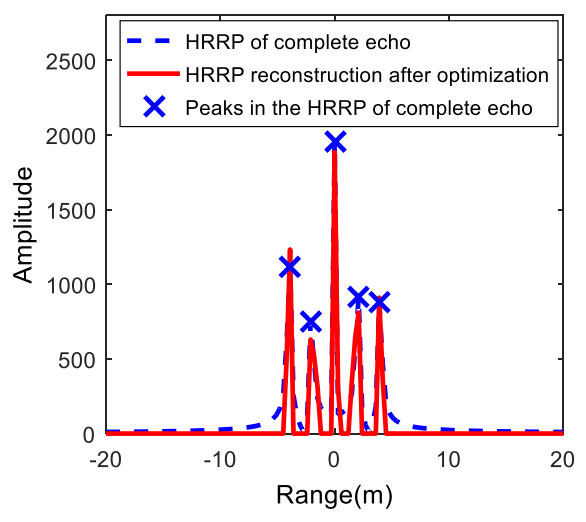

(b)

Fig. 6 The HRRP reconstruction comparison when $T_{S_{n}} \sim[0.5 \mu \mathrm{s}, 1.2 \mu \mathrm{s}]$. a HRRP reconstruction before optimization. b HRRP reconstruction after optimization 


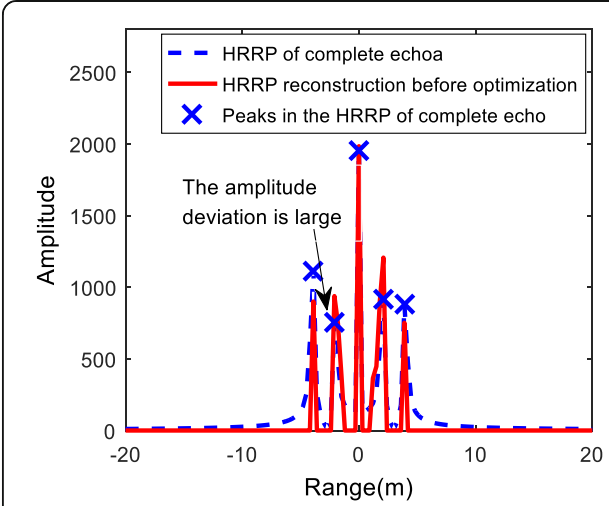

(a)

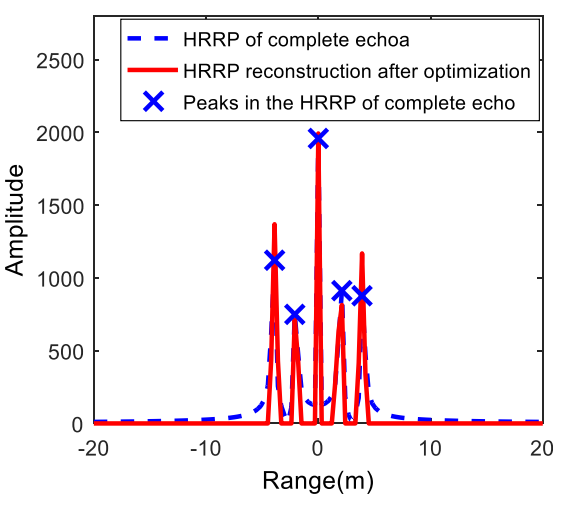

(b)

Fig. 7 The HRRP reconstruction comparison when $T_{S_{n}} \sim[0.8 \mu \mathrm{s}, 1.1 \mu \mathrm{s}]$. a HRRP reconstruction before optimization. b HRRP reconstruction after optimization

optimized and non-optimized R-ITR period sequences have good RIP property when $T_{S_{n}} \sim[0.5 \mu \mathrm{s}, 0.8 \mu \mathrm{s}]$.

Specially, the HRRP reconstruction comparisons are presented in Fig. 6 when $T_{S_{n}} \sim$ [0.5 $\mu \mathrm{s}, 1.2 \mu \mathrm{s}]$. Figure $6 \mathrm{a}$ is the reconstructed HRRP versus the HRRP of the complete echo. Figure $6 \mathrm{~b}$ is the reconstructed HRRP obtained by the optimized R-ITR period sequence. It can be found that the deviation between the reconstructed HRRP and the HRRP of the complete echo is smaller in Fig. 6b than that in Fig. 6a. For example, the amplitude of the scattering center 5 in the reconstructed HRRP in Fig. $6 \mathrm{~b}$ is closer to the HRRP of the complete echo. Therefore, the results indicate the effectiveness of the optimization method.

When $T_{s_{n}} \sim[0.8 \mu \mathrm{s}, 1.1 \mu \mathrm{s}]$, the HRRP comparison is obtained in Fig. 7. Because there are many large ITR periods, the columns cross-correlation coefficients are big. And the HRRP reconstruction performance is poor as shown in Fig. 7a. However, the R-ITR period sequence is optimized after GA; the reconstructed HRRP is presented in Fig. $7 \mathrm{~b}$. It can be found that the deviation between the reconstructed HRRP and the HRRP of the complete echo is small which indicates the good RIP of the sensing matrix after optimization.

In order to analyze the HRRP reconstruction performance, the normalized error between the reconstructed HRRP and the HRRP of complete echo is defined as:

$$
\text { Error }=\left\|\mathbf{S}_{f}-\overline{\mathbf{S}_{f}}\right\|_{2} /\left\|\mathbf{S}_{f}\right\|_{2}
$$

where $\mathbf{S}_{f}$ is the HRRP of complete echo, and $\overline{\mathbf{S}_{f}}$ is the reconstructed HRRP.

For different R-ITR period sequences, the normalized errors are summarized in Table 5.

Table 5 The normalized error between the reconstructed HRRP and the real HRRP before and after optimization

\begin{tabular}{llll}
\hline & $T_{s_{n} \in[0.5 \mu \mathrm{s}, 0.8 \mu \mathrm{s}]}$ & $T_{s_{n}} \in[0.5 \mu \mathrm{s}, 1.2 \mu \mathrm{s}]$ & $T_{s_{n} \in[0.8 \mu \mathrm{s}, 1.1 \mu \mathrm{s}]}$ \\
\hline Before optimization & 0.2887 & 0.3441 & 0.4463 \\
PSO-based optimization method & 0.2573 & 0.2930 & 0.4027 \\
Proposed optimization method & 0.2562 & 0.2833 & 0.3130 \\
\hline
\end{tabular}




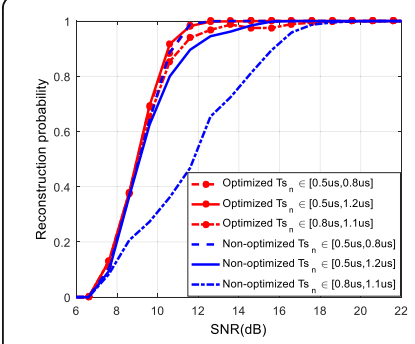

(a)

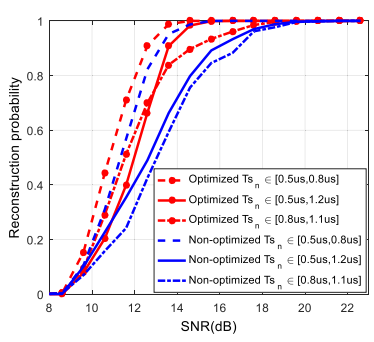

(b)

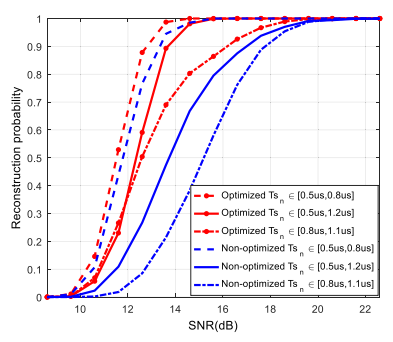

(c)

Fig. 8 Reconstruction probabilities of scattering 1 and 2 and HRRP. a Reconstruction probabilities of scattering 1. b Reconstruction probabilities of scattering 2. $\mathbf{c}$ Reconstruction probabilities of HRRP

In Table 5, the normalized error of HRRP after optimization is smaller than that before optimization. The normalized error is the smallest when $T_{S_{n}} \sim[0.5 \mu \mathrm{s}, 0.8 \mu \mathrm{s}]$, and it decreases to 0.3130 when $T_{S_{n}} \sim[0.8 \mu \mathrm{s}, 1.1 \mu \mathrm{s}]$. Besides, the normalized error obtained by the PSO is larger than the proposed method as well. Therefore, it indicates that the proposed optimization method based on GA is effective to improve the HRRP reconstruction performance.

\subsection{Reconstruction probabilities of scattering centers and HRRP based on GA}

When the residual error between the reconstructed and original information is small enough, the reconstruction can be assumed to be correct [4]. Therefore, the HRRP can be supposed as corrected when the peaks $P_{\text {os }}$ (peaks) is smaller than the range cell, and the normalized amplitude deviation $A_{\text {dev }}$ (peaks) is smaller than the threshold:

$$
\left\{\begin{array}{l}
P_{\text {os }}(\text { peaks }) \leq R_{r} \\
A_{\text {dev }}(\text { peaks }) \leq \gamma
\end{array}\right.
$$

where $R_{r}$ is the range resolution, and $R_{r}=0.3 m$ as the bandwidth is $500 \mathrm{MHz} . \gamma$ is the threshold which is set as 0.5 .

The output signal-to-noise ratio (SNR) is defined as:

$$
\mathrm{SNR}=\max \left(\left|A_{s w}\right|^{2}\right) / \overline{\left|A_{n}\right|^{2}}
$$

where $A_{s w}$ is the peak value in target range cell, and $\overline{\left|A_{n}\right|^{2}}$ is the noise value which is determined by $\boldsymbol{\xi}$.

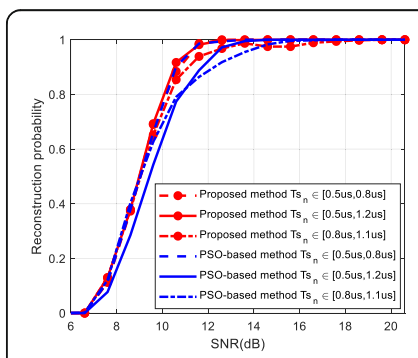

(a)

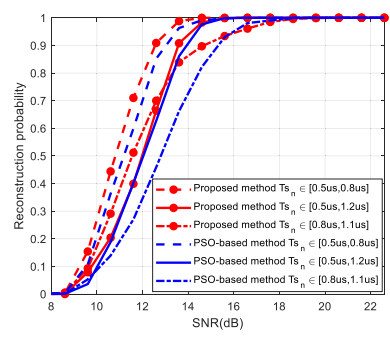

(b)

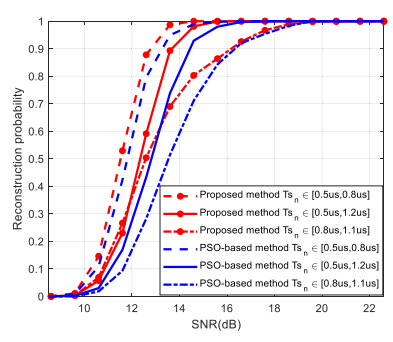

(c)

Fig. 9 Reconstruction probability comparison between the proposed method and the PSO method. a Comparison of scattering 1 reconstruction probabilities. $\mathbf{b}$ Comparison of scattering 2 reconstruction probabilities. c Comparison of HRRP reconstruction probabilities 
The reconstruction probabilities of scattering 1 and 2 and HRRP are presented in Fig. 8. Figure $8 \mathrm{a}, \mathrm{b}$ are the reconstruction probabilities of scattering center 1 and 2 before and after optimization. Because the coefficient of scattering center 1 is bigger than scattering center 2, the reconstruction probability is better. Meanwhile, the reconstruction probability is improved after GA optimization which is in accordance with the HRRP reconstruction error in Table 5.

When all the scattering centers are reconstructed, the HRRP can be supposed as success. Then, the HRRP reconstruction probability is presented in Fig. 8c. Similarly, the reconstruction probability is better after the optimization of R-ITR period sequence. Besides, the best reconstruction probability can be obtained with the R-ITR periods of $T_{s_{n}} \sim[0.5 \mu \mathrm{s}, 0.8 \mu \mathrm{s}]$.

The reconstruction probability comparsion between the proposed method and the PSO method are conducted and presented in Fig. 9.

Figure 9a, b are the reconstruction probability comparison of scattering 1 and scattering 2. It can be found that the reconstruction probabilities obtained by the proposed method are bigger than those obtained by the PSO-based method. The HRRP reconstruction is successful when the five peaks of the HRRP is recovered. Therefore, the HRRP reconstruction probability of the proposed method is better compared with the PSO-based method as shown in Fig. 9c.

\section{Conclusion}

The R-ITR period sequence optimization method is proposed in this paper based on GA. The columns cross-correlation coefficient is used to evaluate the fitness value of the population in GA. Then, the HRRP reconstruction probabilities are obtained based on the proposed optimized ITR period sequence, the PSO ITR period sequence, and the ITR period sequence before optimization. Simulation and comparison results show that the columns cross-correlation coefficient is minimized after optimization which indicates the effectiveness of the proposed method. The optimization method of the ITR period sequence can be used in the radar signal design in the future.

Abbreviations

ITR: Interrupted transmitting and receiving; R-ITR: Randomly interrupted transmitting and receiving; HRRP: Highresolution range profile; CS: Compressive sensing; RIP: Restricted isometry property; GA: Genetic algorithm; RFS: Radio frequency simulation

Acknowledgements

Not applicable.

Authors' contributions

Methodology: X.L. Validation: F.Z. and X.L. Formal analysis: F.Z. and X.A. Investigation: X.A. Writing-original draft preparation: X.L. and Q.W. The authors read and approved the final manuscript.

Funding

This research was funded by the National Natural Science Foundation of China, grant numbers 61890542 and 62001481.

Availability of data and materials

All data generated or analyzed during this study are included in this published article.

Ethics approval and consent to participate

Not applicable.

Consent for publication

Not applicable. 
Competing interests

The authors declare that they have no competing interests.

Received: 28 October 2020 Accepted: 22 January 2021

Published online: 04 February 2021

\section{References}

1. W. He, L. Zhang and N. Li, "A new method to improve precision of target position in RFS," in International Conference on Microwave and Millimeter Wave Technology, Guilin, 2007, pp. 1-3.

2. J. Liu, G. Li, L. Ma et al., "Dynamic measurement of micro-motion targets in microwave anechoic chamber," in International Radar Conference, 2009, no. 551, pp. 1-4.

3. X. Liu, J. Liu, F. Zhao, et al., An equivalent simulation method of pulse radar measurement in anechoic chamber. IEEE Geosci. Remote Sens. Lett. 14(7), 1081-1085 (2017)

4. J.A. Tropp, A.C. Gilbert, Signal recovery from random measurements via orthogonal matching pursuit. IEEE Trans. Inf. Theory 53(12), 4655-4666 (2007)

5. S. Wei, L. Zhang, H. Ma, H. Liu, Sparse frequency waveform optimization for high-resolution ISAR imaging. IEEE Trans. Geosci. Remote Sens. 58(1), 546-566 (2020)

6. L. Yan, P. Addabbo, C. Hao, D. Orlando, A. Farina, New ECCM techniques against noiselike and/or coherent interferers. IEEE Trans. Aerosp. Electron. Syst. 56(2), 1172-1188 (2020)

7. L. Yan, P. Addabbo, Y. Zhang, C. Hao, J. Liu, J. Li, D. Orlando, A sparse learning approach to the detection of multiple noise-like jammers. IEEE Trans. Aerosp. Electron. Syst. 56(6), 4367-4383 (2020)

8. P. Addabbo, A. Aubry, A. De Maio, L. Pallotta, S. Ullo, HRR profile estimation using SLIM. IET Radar, Sonar Navig. 13(4), 512-521 (2019)

9. X. Liu, J. Liu, F. Zhao, X. Ai, G. Wang, A novel strategy for pulse radar HRRP reconstruction based on randomly interrupted transmitting and receiving in radio frequency simulation. IEEE Trans. Antennas Propag. 66(5), 2569-2580 (2018)

10. E.J. Candes, The restricted isometry property and its implications for compressed sensing. Acad. Sci. 1(346), 589-592 (2008)

11. Z. Liu, X. Wei, X. Li, Aliasing-free moving target detection in random pulse repetition interval radar based on compressed sensing. IEEE Sensors J. 13(7), 2523-2534 (2013)

12. J. Bourgain, S.J. Dilworth, K. Ford, S. Konyagin, D. Kutzarova, Explicit constructions of RIP matrices and related problems. J. Math. Duke 159, 145-185 (2010)

13. Y. Wang, F. Xi and S. Chen, "Structure-based sensing matrix optimization for extended target ranging," 2020 IEEE Radar Conference (RadarConf20), Florence, Italy, 2020, pp. 1-5.

14. B. Biswal, T. K. Panda, S. Hasan, P. K. Dash and B. K. Panigrahi, "Nonstationary power signal processing and pattern recognition using genetic algorithm," 2007 6th International Conference on Information, Communications \& Signal Processing, Singapore, 2007, pp. 1-5.

15. K. L. Holladay and K. A. Robbins, "Evolution of signal processing algorithms using vector based genetic programming," 2007 15th International Conference on Digital Signal Processing, Cardiff, 2007, pp. 503-506.

16. X. Jia, G. Lu, A hybrid Taguchi binary particle swarm optimization for antenna designs. IEEE Antennas Wirel. Propag. Lett. 18(8), 1581-1585 (2019)

17. M. Jamil and H. Zepernick, "Sequence design for radar applications using particle swarm optimization," 2016 International Conference on Advanced Technologies for Communications (ATC), Hanoi, 2016, pp. 210-214

\section{Publisher's Note}

Springer Nature remains neutral with regard to jurisdictional claims in published maps and institutional affiliations.

\section{Submit your manuscript to a SpringerOpen ${ }^{\circ}$ journal and benefit from:}

- Convenient online submission

- Rigorous peer review

- Open access: articles freely available online

- High visibility within the field

- Retaining the copyright to your article

Submit your next manuscript at $\boldsymbol{\Delta}$ springeropen.com 